\title{
Representation in the Human Brain of Food Texture and Oral Fat
}

\author{
Ivan E. de Araujo ${ }^{1,2}$ and Edmund T. Rolls ${ }^{1,2}$ \\ ${ }^{1}$ Department of Experimental Psychology, University of Oxford, Oxford OX1 3UD, United Kingdom, and ${ }^{2}$ Centre for Functional Magnetic Resonance \\ Imaging of the Brain, John Radcliffe Hospital, Oxford OX3 9DU, United Kingdom
}

\begin{abstract}
Important factors that influence food palatability are its texture and fat content. We investigated their representation in the human brain using event-related functional magnetic resonance imaging. It was shown that the viscosity of oral stimuli is represented in the (primary) taste cortex in the anterior insula, in which activation was proportional to the log of the viscosity of a cellulose stimulus (carboxymethyl cellulose), and was also produced by sucrose. Oral viscosity was also represented in a mid-insular region that was posterior to the taste cortex. Third, it was found that oral delivery of fatty vegetable oil activates both of these insular cortex regions, the hypothalamus, and the dorsal midanterior cingulate cortex. Fourth, it was found that the ventral anterior cingulate cortex, where it borders the medial orbitofrontal cortex, was activated by oral fat independently of its viscosity and was also activated by sucrose taste. This ventral anterior cingulate region thus represents two indicators of the energy content and palatability of foods. These are the first investigations of the oral sensory representation of food texture and fat in the human brain, and they start to reveal brain mechanisms that may be important in texture-related sensory properties of foods that make them palatable and that may accordingly play a role in the hedonic responses to foods, the control of food intake, and obesity.
\end{abstract}

Key words: cingulate; food; insula; obesity; reward; taste

\section{Introduction}

It is important to understand the representation in the brain of fat as well as other textural properties of food that influence its palatability. Fat texture can increase the palatability of foods and is also a major cue about the energy density of foods (Drewnowski, 1998). Fat is frequently overconsumed in palatable high-energy density foods, and the overeating of these foods is common in obese humans (McCrory et al., 1999; Rolls, 2000). It is thus of interest to determine what is being sensed orally that signals that fat is present in a food and the brain areas that represent these oral signals. This will pave the way toward understanding the brain areas where the palatability of oral fat is represented, how different satiety signals affect this representation, and how these processes may differ in obese humans. Understanding the factors that contribute to obesity is of clinical importance, given the increasing prevalence of obesity, and the health risks, including those arising from coronary heart disease and diabetes that are associated with obesity (Criqui et al., 1993).

Very little is known of the representation of oral texture, including oral fat in the human sensory pathways and brain. Studies have been performed at the single-neuron level in non-human primates to provide a basis for understanding the taste, texture, and fat systems in humans (Rolls, 1997, 1999). The primary taste

Received Nov. 28, 2003; revised Feb. 10, 2004; accepted Feb. 10, 2004.

This research was supported by the Medical Research Council.

Correspondence should be addressed to Prof. Edmund T. Rolls, University of Oxford, Department of Experimental

Psychology, South Parks Road, 0xford 0X1 3UD,UK. E-mail: edmund.rolls@psy.ox.ac.uk.

DOI:10.1523/JNEUROSCI.0130-04.2004

Copyright $\odot 2004$ Society for Neuroscience $\quad$ 0270-6474/04/243086-08\$15.00/0 cortex in the anterior insular/frontal opercular area is activated not only by taste stimuli (Scott et al., 1986; Yaxley et al., 1990) but also by oral texture as manipulated by a texture series made from the odorless and tasteless thickening agent carboxymethyl cellulose (CMC) (E. T. Rolls, M. Kadohisa, and J. V. Verhagen, unpublished observations). In the primate secondary taste cortex within the orbitofrontal cortex, some neurons respond to the viscosity of food, to taste, or to both viscosity and taste (Rolls et al., 2003b). Moreover, there is a separate representation of fat in the mouth provided by orbitofrontal cortex neurons, and this representation is provided by a texture, not chemical, property of the fat, in that these neurons are activated not only by fatty oils but also by nonfatty oils, such as silicone oil and mineral oil (pure hydrocarbon) (Rolls et al., 1999). Furthermore, this representation of fat is independent of viscosity, in that these fat-texture neurons may not respond to cellulose-based viscosity stimuli (Verhagen et al., 2003).

This investigation was performed to provide the first evidence on the representation of oral texture and fat in the human brain. Previous imaging studies in humans have shown that taste is represented in the anterior insular and frontal opercular region and in the orbitofrontal cortex, which is putatively the human secondary taste cortex (Francis et al., 1999; Small et al., 1999; O'Doherty et al., 2001; de Araujo et al., 2003a,b). Furthermore, taste and olfactory modalities in humans converge in the orbitofrontal cortex and adjoining far anterior, agranular, insula (de Araujo et al., 2003c; Rolls et al., 2003a).

\section{Materials and Methods}

The design of the event-related functional magnetic resonance imaging (fMRI) study described here was to measure the neural effects of the 
intraoral delivery of a tasteless and odorless stimulus, carboxymethyl cellulose, at three different viscosity coefficients; one high-fat content stimulus (vegetable oil) and a unimodal taste stimulus (sucrose). The first aim was to reveal the areas of the human brain that represent oral viscosity. The second aim was to investigate which of these texturerelated areas also responded to taste. Third, we aimed to reveal which areas respond to the sensory properties of fat in the mouth, including the characterization of the regions responding to fat independently of viscosity. Fourth, we aimed to measure where in the brain the activation correlated with subjective ratings given during the experiment of the texture (thickness), fat content, and sweetness. The control for all of the stimuli was a tasteless solution with approximately the ionic components of saliva.

Subjects. Twelve healthy right-handed subjects (of whom seven were males; age range, 23-41 years) participated in the study. Written informed consent from all subjects and ethical approval were obtained before the experiment. The subjects were mildly hungry, having not eaten a meal in the preceding $3 \mathrm{hr}$.

Stimuli. Three viscosity stimuli, one high-fat content stimulus, and a taste stimulus provided the basic set. A viscosity series was produced with carboxymethyl cellulose (CMC 7MF Blanose; Hercules, Wilmington, $\mathrm{DE})$, a tasteless and odorless thickening agent commonly found in commercially produced foods. CMC was prepared in a tasteless solution (containing the main ionic components of saliva, $25 \mathrm{~mm} \mathrm{KCl}$ and $2.5 \mathrm{~mm}$ $\mathrm{NaHCO}_{3}$ in distilled water) (de Araujo et al., 2003a). With respect to $\mathrm{CMC}$, the term "apparent viscosity" is used to indicate that this compound does not behave rheologically as a Newtonian fluid, showing thinning behavior as shear forces increase (Verhagen et al., 2003), and that the viscosity measurement is taken at a particular shear rate. Viscosity was measured using a calibrated Brookfield rotary viscometer (type LVT; Brookfield Engineering Laboratories, Middleboro, MA) at $60 \mathrm{rpm}$ (shear rate, $\sim 12 \mathrm{sec}^{-1}$ ) and at $23^{\circ} \mathrm{C}$. The viscosity values for the viscosity stimuli were 1 centipoise $(\mathrm{cP})$ (in this case, the tasteless solution with no CMC added), $\mathrm{CMC}$ at $50 \mathrm{cP}$, and $\mathrm{CMC}$ at $1000 \mathrm{cP}$ (the corresponding concentrations were 11.4 and $29.3 \mathrm{gm} / \mathrm{l}$ ). [Note that $1 \mathrm{cP}$ is equivalent to 1 $\mathrm{Pa} / \mathrm{sec}^{-1}$. For those not familiar with viscosity values, it may be helpful to note that the viscosity of water at $20^{\circ} \mathrm{C}$ is $\sim 1 \mathrm{cP}$, of the corn oil used for cooking is typically $50-60 \mathrm{cP}$, and of treacle (known in the United States as Blackstrap molasses) is typically $5000-10,000 \mathrm{cP}$.] The fat stimulus was vegetable oil (rapeseed oil consisting of $6.1 \mathrm{gm}$ of saturate fat, $54.4 \mathrm{gm}$ of monounsaturated fat, and 26.9 gm of polyunsaturated fat per $100 \mathrm{ml}$; Sainsbury's Supermarkets, UK) with a measured viscosity in the above conditions of $50 \mathrm{cP}$. This oil was chosen because it was the most odorless and tasteless of those that could be obtained. The taste stimulus was $1 \mathrm{M}$ sucrose (Sigma, Poole, UK) (which has a viscosity of $\sim 2 \mathrm{cP}$ ). The tasteless solution was used as a control stimulus for the effects of the somatosensory effects produced by introducing a liquid with a viscosity of $1 \mathrm{cP}$ into the mouth and making the single tongue movement required to distribute the fluid throughout the oral cavity. The stimuli and concentrations were chosen on the basis of psychophysical tests performed on a panel of subjects before the scanning study.

Experimental design. The experimental protocol consisted of an eventrelated interleaved design using in random order the five stimuli that consisted of the tasteless solution, CMC prepared in tasteless solution (at 50 and $1000 \mathrm{cP}$ ), vegetable oil (at $50 \mathrm{cP}$ ), and sucrose. Stimuli were delivered to the subject's mouth through seven polythene tubes that were held between the lips. Each tube of $\sim 1 \mathrm{~m}$ in length was connected to a separate reservoir via a syringe and a one-way syringe valve (Fisher Scientific, Loughborough, UK), which allowed $0.75 \mathrm{ml}$ of any stimulus to be delivered manually under computer instruction.

At the beginning of each stimulus delivery, one of the five stimuli chosen by random permutation was delivered in $0.75 \mathrm{ml}$ aliquots to the subject's mouth. Swallowing was cued by a visual stimulus after $10 \mathrm{sec}$ (after initial instruction and training). After a delay of $3 \mathrm{sec}$, the subject was asked to rate each of the stimuli for thickness, fat content, and sweetness by using three separate visual analog rating scales anchored at 0 for very fluid-no fat content-no sweet taste and +4 for very thick-very fatty-very sweet. Each rating period was $5 \mathrm{sec}$ long. The tasteless solution containing the main ionic components of saliva $(25 \mathrm{~mm} \mathrm{KCl}$ plus $2.5 \mathrm{~mm}$
$\mathrm{NaHCO}_{3}$ ) was then administered in exactly the same way as the test stimulus, and the subject was cued to swallow again after $10 \mathrm{sec}$. Then there was a 3 sec delay period allowed for swallowing, followed by a $1 \mathrm{sec}$ gap until the start of the next trial. This taste trial was repeated for each of the six stimuli, and the whole cycle was repeated nine times.

$f M R I$ data acquisition. Images were acquired with a $3 \mathrm{~T}$ Varian/Siemens whole-body scanner at the Centre for Functional Magnetic Resonance Imaging at Oxford, where $14 \mathrm{~T}^{\star}{ }^{\star}$ weighted echo planar imaging (EPI) slices were acquired every $2 \mathrm{sec}$ (repetition time, $2 \mathrm{sec}$ ). We used a set of optimizing techniques to select the imaging parameters to minimize susceptibility and distortion artifact in the orbitofrontal cortex, as described in detail by Wilson et al. (2002) and in previous publications from this laboratory. The relevant factors include imaging in the coronal plane, minimizing voxel size in the plane of the imaging, as high a gradient switching frequency as possible $(960 \mathrm{~Hz})$, a short echo time of 25 $\mathrm{msec}$, and local shimming for the inferior frontal area.

The matrix size was $64 \times 64 \mathrm{~mm}$, and the field of view was $192 \times 192$ $\mathrm{mm}$. Continuous coverage was obtained from +60 (anteroposterior) to -38 (anteroposterior). Acquisition was performed during the task performance yielding 1008 volumes in total. A whole-brain $\mathrm{T} 2^{\star}$ weighted EPI volume of the above dimensions and an anatomical T1 volume with slice thickness $1.5 \mathrm{~mm}$ and in-plane resolution of $1.5 \times 1.5 \mathrm{~mm}$ was also acquired.

fMRI data analysis. The imaging data were analyzed using SPM2 (Wellcome Department of Imaging Neuroscience, University of London, London, UK). Pre-processing of the data used SPM2 for realignment, reslicing with generalized interpolation (Thévenaz et al., 2000), normalization to MNI coordinate system (Montreal Neurological Institute) (Collins et al., 1994), and spatial smoothing with a $10 \mathrm{~mm}$ full-width at half-maximum isotropic Gaussian kernel and global scaling. Time series nonsphericity at each voxel was estimated and corrected for (Friston et al., 2002), and a high-pass filter with a cutoff period of $156 \mathrm{sec}$ was applied.

A general linear model was then applied to the time course of activation in which stimulus onsets were modeled as single impulse response functions and then convolved with the canonical hemodynamic response function (Friston et al., 1994). Time and dispersion derivatives were included in the basis functions set. After smoothness estimation (Kiebel et al., 1999), linear contrasts of parameter estimates were defined to test the specific effects of each condition with each individual dataset. To assess the effects of each stimulus, comparisons were defined between each of the stimuli and the corresponding tasteless $1 \mathrm{cP}$ control stimulus within a trial. This procedure ensured that all the simple effect comparisons consisted of a set of orthogonal linear contrasts.

Voxel values for each contrast resulted in a statistical parametric map of the corresponding $t$ statistic, which was then transformed into the unit normal distribution (SPMZ). The statistical parametric maps from each individual dataset were then entered into second-level, random-effects analyses accounting for both scan-to-scan and subject-to-subject variability. More precisely, the sets of individual statistical maps corresponding to a specific effect of interest (i.e., to within-subjects differential effects) were entered as covariates in multiple regression models (without a constant) as implemented in SPM2, and the corresponding group effects were assessed by applying linear contrasts (again after smoothness estimation) to the (second-level) parameter estimates generating a $t$ statistics map for each group effect of interest. The above allowed us to perform conjunction analyses (Friston et al., 1999) at the second level. When appropriate, correlation analyses of the fMRI blood oxygenation level-dependent (BOLD) signal with given parameters of interest (e.g., viscosity measures) were performed at the second-level through applying one-sample $t$ tests to the first-level $t$ maps resulting from performing linear parametric modulation as implemented in SPM2.

Reported $p$ values based on this group analysis are either corrected for the number of comparisons (resels) in the entire volume ("whole-brain" multiple comparisons) (Worsley et al., 1996) or controlled for false discovery rate (FDR correction) (Genovese et al., 2002). For a priori regions of interest (e.g., the insular, anterior cingulate, and orbitofrontal cortices), reported $p$ values were corrected for the number of comparisons made within each region [small volume correction (SVC)] (Worsley et 
al., 1996). Checks were performed that the results were not influenced by motion artifact by rerunning the analyses using the estimated motion parameters as covariates of no interest in the design matrix and confirming that the results were unaffected.

\section{Results}

\section{Behavioral data}

First, the subjective ratings for stimulus thickness given by the subjects revealed a significant correlation between these ratings and the logarithm of the viscosity (Mela et al., 1994; Theunissen and Kroeze, 1995): Pearson's $r=0.67 ; p<0.001$. Second, significant correlations were found between the fat content subjective ratings and the logarithm of the viscosity $(r=$ $0.51 ; p<0.001)$ and, importantly, between fat content subjective ratings and thickness subjective ratings $(r=0.75 ; p<$ 0.0001). The above thus shows that, first, subjects were able to efficiently discriminate between different viscosities and, second, that increased viscosity was a major determinant in perceived fat content. [The subjective thickness ratings for fat (which had a viscosity of $50 \mathrm{cP}$ ), CMC at $1000 \mathrm{cP}$, $\mathrm{CMC}$ at $50 \mathrm{cP}$, tasteless solution, and sucrose were, respectively (mean \pm SEM) as follows: $1.8 \pm 0.1,2.8 \pm 0.1,1.31 \pm 0.08$, $0.95 \pm 0.08$, and $0.87 \pm 0.07$, and the subjective fat ratings for these stimuli were $2.2 \pm 0.1,2.3 \pm 0.12,1.56 \pm 0.09,1.07 \pm$ 0.09 , and $0.55 \pm 0.05]$.

Finally, subjects efficiently differentiated between the unimodal taste stimulus ( $1 \mathrm{~m}$ sucrose) and each of the other stimuli: the subjective sweetness rating for sucrose was (mean \pm SEM) $2.86 \pm 0.08$, whereas for fat, CMC at $1000 \mathrm{cP}$, $\mathrm{CMC}$ at $50 \mathrm{cP}$, and tasteless solution, the sweetness ratings were, respectively, $0.24 \pm 0.03,0.27 \pm 0.04,0.32 \pm 0.03$, and $0.31 \pm$ 0.03 . Pairwise one-tailed comparisons between the sweetness ratings for sucrose and for the other stimuli are each significant at $p<0.0001$.

The viscosity (carboxymethyl cellulose) and fat (vegetable oil) stimuli were selected for use in this investigation to be as tasteless and odorless as possible. To confirm that they were odorless and tasteless, we performed psychophysical investigations with eight subjects (six of whom were subjects in the neuroimaging experiments), providing subjective ratings of the intensity of the stimuli on a 100 visual analog rating scale labeled at one end "very weak" and at the other end "very intense" (for psychophysical methods, see Rolls et al., 1983). The stimuli were delivered intraorally in aqueous solution in $0.75 \mathrm{ml}$ aliquots. The intensity of the taste of the $50 \mathrm{cP} C M C$ was rated very low $(13 \pm 4 \mathrm{~mm}$; mean $\pm \mathrm{SEM})$ from the "weak" end of the scale and so was the vegetable oil $(14 \pm 4 \mathrm{~mm})$ compared with $17 \pm 6 \mathrm{~mm}$ for water and $68 \pm 6$ $\mathrm{mm}$ for $1 \mathrm{M}$ sucrose taste. The intensity of the odor of the carboxymethyl cellulose was rated very low $(11 \pm 4) \mathrm{mm}$ from the "weak" end of the scale and so was the vegetable oil $(8 \pm 3 \mathrm{~mm})$ compared with $12 \pm 6 \mathrm{~mm}$ for water and $43 \pm 7 \mathrm{~mm}$ for the same strawberry odor used in the neuroimaging investigation by de Araujo et al. (2003c), in which it was shown to activate olfactory cortical areas. The intensities of the taste and odor of both the carboxymethyl cellulose and the vegetable oil were not significantly different from those of water $(p>0.09)$ and were significantly different from sucrose taste $(p<0.001)$ and from strawberry odor $(p<0.003)$ [as shown by Bonferroni corrected post hoc $t$ tests after a significant $(p<0.001)$ one-way ANOVA for taste and separately for odor].

\section{fMRI data}

\section{Correlation of the BOLD signal with viscosity}

A correlation analysis was performed between the fMRI BOLD signal and the viscosity of the stimuli to reveal the human brain areas representing the viscosity of intraoral stimuli (see Materials and Methods). Significant correlations were found in a midposterior region of the left insular cortex [MNI coordinates $(-48$, $-12,6) ; Z=6.06 ; p<0.05$, corrected for multiple comparisons]. This activation (and related raw data analysis) is shown in Figure 1, top row. A more anterior part of the left insula (probably including part of the putative human primary taste cortex) was also significantly activated $[(-36,14,8]) ; Z=4.6 ; p<0.05$, corrected for multiple comparisons). This activation (and related raw data analysis) is shown in Figure 1, bottom row. (The correlation analysis shown was performed across the three stimuli in the CMC viscosity series, and closely comparable results were obtained if the analysis was performed over the whole set of stimuli).

At lower thresholds, significant correlations with stimulus viscosity are found in this same midposterior insular region in the 

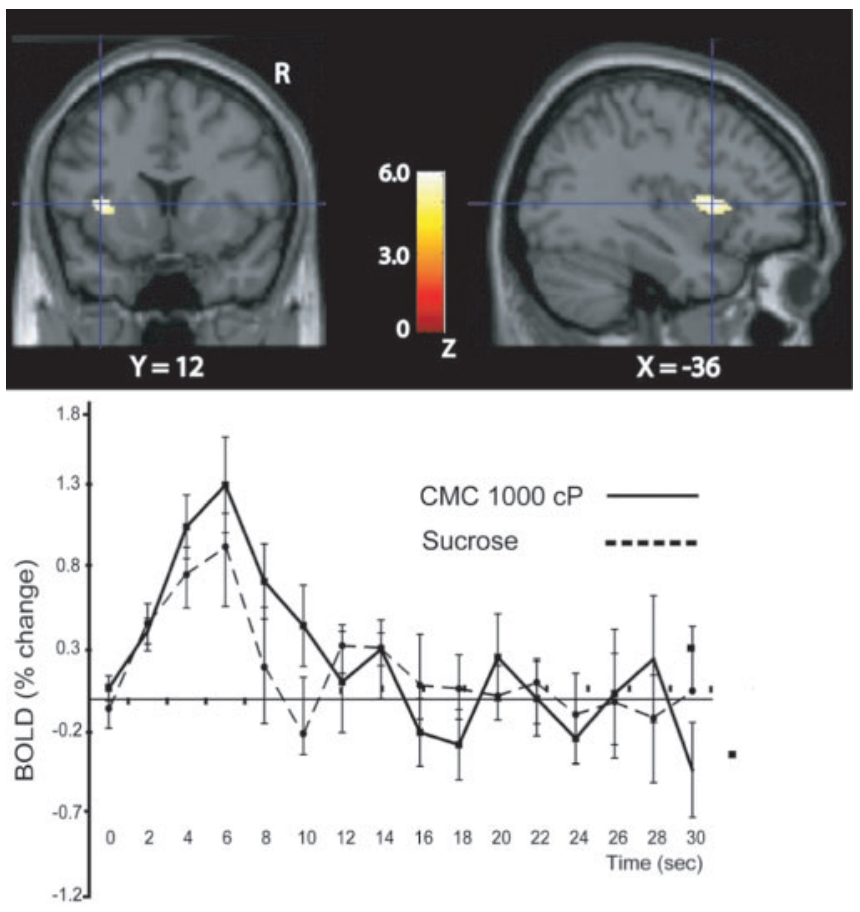

Figure 2. Top, Anterior insula activation by (CMC at $1000 \mathrm{CP}$ - control) and (sucrose control), as revealed by conjunction analysis. The sagittal slice on the right shows the anteroposterior extent of this activation. Bottom, Average time courses (across trials and subjects) for CMC at $1000 \mathrm{CP}$ and sucrose from the anterior insula voxel indicated by the crosshairs. The time course data reveal a robust response for both types of stimuli (i.e., unimodal oral somatosensory and unimodal taste) in this part of the human insula. The image was thresholded at $p<0.0001$, uncorrected to show the extent of the activation. R, Right.

right hemisphere $[(48,-8,12) ; Z=3.9 ; p<0.0003$, FDR corrected; see Materials and Methods]. Also, correlations were found in the right hemisphere of the anterior insular cortex [ 36 , $10,10) ; Z=3.53 ; p<0.0001$, uncorrected).

Regions activated by both unimodal texture and taste stimuli Significant activations were found in the anterior part of the insular cortex to both viscosity and taste stimuli. In fact, a conjunction analysis (see Materials and Methods) across the conditions [(CMC at $1000 \mathrm{cP}-$ control) and (sucrose - control)] revealed a significant activation in the anterior part of the insular cortex, which included the anterior insular region in which a correlation was found with viscosity $[(-36,12,6) ; Z=4.5$; $p<0.03$, corrected for multiple comparisons). This activation and the group average time courses for both conditions are shown in Figure 2. Lowering the threshold to $p<0.0001$ uncorrected reveals that this conjunctive activation by taste and viscosity extends in the anteroposterior direction from $Y=26$ to $Y=0$, indicating thus that an extensive part of the human taste insula responds to oral somatosensory stimuli. The part of the mid-insula that responded to viscosity but not taste was located at approximately $Y=-8$ to $Y=-14$. This is thus an oral somatosensory part of the insula without taste inputs.

\section{Responses to the oral delivery of fat}

The oral delivery of fat [as assessed by the comparison (fat control)] elicits highly significant responses in the left mid-insula $[(-36,0,-1) ; Z=5.16 ; p<0.01$, corrected for (family-wise) multiple comparisons $]$ and left anterior insula $[(-36,18,4) ; Z=$ $4.85 ; p<0.05$, corrected for multiple comparisons]. These activations are shown in Figure 3, top row, left and middle. Lowering the threshold to $p<0.0001$ uncorrected reveals activations bilaterally in the insula with an anteroposterior extent from $Y=26$ (at the transition zone with the caudal part of the orbitofrontal cortex) to $Y=-13$, including voxels in the adjacent opercular cortex. Conjunction analysis (see Materials and Methods) across the conditions [(CMC at $50 \mathrm{cP}-$ control) and (fat - control)] indicates that most responses to fat found in the insular cortex are in the same region as the responses driven by viscosity. Significant activations for this conjunction were found throughout the insula, with peaks in the left mid-insula $[(-38,2,-6) ; Z=4.10$; $p<0.03$, FDR corrected), extending posteriorly to $Y=-2$, left anterior insula $[(-28,14,-6) ; Z=4.21 ; p<0.03$, FDR corrected], and in the adjacent right lateral orbitofrontal cortex [ $(28$, $30,-10) ; Z=3.75 ; p<0.04$, FDR corrected]. Average time course data (across trials and subjects) from the mid-insular cortex (at $Y=-1)$ for conjunctive activations for fat and CMC at 50 $\mathrm{cP}$ are shown in Figure 3. Thus, the insular region activated by fat extends from the taste cortex anteriorly through to the midinsular oral somatosensory nontaste region.

Additional significant activations were observed in the hypothalamic region $[(12,0,-4) ; Z=4.06 ; p<0.03$, FDR corrected] (Fig. 3, top row, left) and in the anterior cingulate cortex, including its more dorsal [Brodmann area (BA) 24; $(4,-8,50) ; Z=$ 5.01; $p<0.05$, corrected for multiple comparisons] and anterior [ventral BA 32; $(-6,40,10) ; Z=4.0 ; p<0.003$, FDR corrected] regions (both activations are shown in Fig. 3, top row, right).

\section{Rostral anterior cingulate cortex activations to both fat} and sucrose

Conjunction analysis (see Materials and Methods) across the conditions (fat - control) and (sucrose - control) reveals a significant activation in the rostral part of the anterior cingulate cortex at its transition zone with the medial part of the orbitofrontal cortex $[(-10,48,-2) ; Z=4.58 ; p<0.05$, corrected for multiple comparisons]. This activation is shown in Figure 4 on the left, and the corresponding average time course data (across trials and subjects) for both conditions are shown on the right of Figure 4.

\section{Activations produced by fat independently of viscosity}

Single-neuron recording studies (Verhagen et al., 2003) indicate that some neurons in the orbitofrontal cortex respond to fat in the mouth independently of viscosity. To test this hypothesis in humans, a correlation analysis (see Materials and Methods) was performed in which the parameter of interest was the stimulus fat content (defined by assigning a value of 1 to the events in which fat was delivered and 0 to all other events), and the viscosity of the stimuli was entered as a covariate. A significant cluster of activation was observed in the rostral part of the anterior cingulate cortex at its transition zone with the medial part of the orbitofrontal cortex $[(-6,44,-2) ; Z=3.78 ; p<0.02$, SVC on the basis of a $20 \mathrm{~mm}$ sphere centered at the peak voxel].

Although there are no previous neuroimaging studies reporting brain activations for fat in the mouth, this region has been found previously to be activated by pleasant taste (de Araujo et al., 2003a) and odor (Rolls et al., 2003a) stimuli and thus was hypothesized a priori to respond to the delivery of a palatable stimulus such as fat. This activation is shown in Figure 5 on the right, and the corresponding adjusted data for the peak voxel is given in the legend to Figure 5 for the conditions fat, $\mathrm{CMC}$ at $50 \mathrm{cP}$, and $\mathrm{CMC}$ at $1000 \mathrm{cP}$, demonstrating that the activation in this region is not fully accounted for by the viscosity component of fat.

A region of interest analysis was performed in the ventral striatum area on the basis of animal studies showing that this 
region is involved in fat intake-related behaviors (Kelley et al., 2002). Activation was found in the left striatum for fat, which is independent of viscosity (i.e., using viscosity as a covariate) $[(-8,16,-2) ; Z=2.52$; $p<0.05$, SVC on the basis of a $10 \mathrm{~mm}$ sphere centered at the peak voxel]. This activation is shown in Figure 5 on the right, and the corresponding adjusted data for the peak voxel is given in the legend to Figure 5 for the conditions fat, $\mathrm{CMC}$ at 50 $\mathrm{cP}$, and $\mathrm{CMC}$ at $1000 \mathrm{cP}$.

To provide additional confirmation that the activation found in the anterior part of the anterior cingulate cortex for the oral delivery of fat is independent of viscosity, the raw data from the peak voxel was extracted from all subjects for additional analysis. First, the time series data from this region did not correlate with the logarithm of the stimuli viscosity $(r=0.2$; $p>0.6)$. Second, an ANOVA revealed significant effects in the time series data for fat content $\left(F_{(1,447)}=12.91 ; p<0.0001\right)$ when viscosity was entered as a covariate. Finally, performing the contrast (fat - CMC at 50 $\mathrm{cP}$ ) also revealed activations in the anterior part of the anterior cingulate cortex, although with a lower level of statistical significance ( $p<0.001$, uncorrected).

\section{Discussion}

First, we found activation of the anterior insular (putative primary) taste cortex of humans by oral viscosity stimuli. Indeed, the BOLD activation here was proportional to the log of the viscosity of the oral stimuli. Fat also activated this region, although not in a way that was identified with the fMRI method as being qualitatively different from the activation produced by a viscosity stimulus made to the same viscosity value with CMC. We hypothesize therefore that the activation of this region corresponds to the details revealed by single-neuron recording in macaques, namely that some neurons in the primary taste cortex are activated by taste unimodally, some by viscosity unimodally, and some by both taste and viscosity (Kadohisa et al., 2003). [In our sample of 59 insular/opercular neurons in two macaques that were activated by taste or oral texture stimuli, $27 \%$ were activated by taste unimodally, some by viscosity unimodally (20\%), and some by both taste and viscosity (24\%).] This neurophysiological evidence shows that the afferents to the primary taste cortex provide taste and somatosensory information by at least partly independent information channels. Other neurons ( $12 \%$ in the insula) are activated in macaques by fat texture independently of viscosity (with, for example, some neurons responding to fat but not to carboxymethyl cellulose stimuli at any viscosity) (Rolls et al., 2003b; Verhagen et al., 2003). The fMRI findings presented here on the human anterior insular cortex are consistent with the hypothesis that the same processing takes place in the human anterior insular cortex.

Second, we found activation of a mid-insular region behind the primary taste cortex that was activated by viscosity and by fat but not by taste (Fig. 1, top left). This may be a purely somatosensory part of the insula that is a higher-order somatosensory cortical area, this part of which is devoted to intraoral somato- sensory inputs. It is known that more posterior to this region in owl monkeys (Jain et al., 2001), the caudal part of the face representation in area $3 \mathrm{~b}$ extends anteriorly beneath the central sulcus and above the upper bank of the lateral sulcus. The representation of the oral cavity is located rostrally to this region extending to the orbitofrontal cortex (Manger et al., 1996; Jain et al., 2001). In humans, tongue movement and stimulation produced activations in a positron emission tomography (PET) study (Grafton et al., 1991) near the central sulcus. In another PET study, Pardo et al. (1997) compared the effects of stimulating either the left or right side of the (protruded) tongue with the effects produced by tongue protrusion only. Significant activations were found contralaterally around the lateral fissure. In contrast, the present study used quantitative variation of the texture of intraoral stimuli by manipulating viscosity and found activation of the midinsular cortex, as well as of the insular taste cortex. This midinsular cortex may represent a range of somatosensory properties of the oral activity because, in a study of the effects of intraoral water, we found that activation in the same mid-insular region was produced by water when thirsty but not after thirst was satiated. We interpreted this as a somatosensory effect related to relief of a dry mouth by water, in that this region was again not activated by taste stimuli (de Araujo et al., 2003b).

Third, we report, also for the first time, activations produced by fat in the mouth. These activations were found in the insular taste cortex and in the mid-insular somatosensory region described above. They were also found in the orbitofrontal cortex, in which some neurons in macaques specifically encode oral fat independently of viscosity (Rolls et al., 2003b; Verhagen et al., 

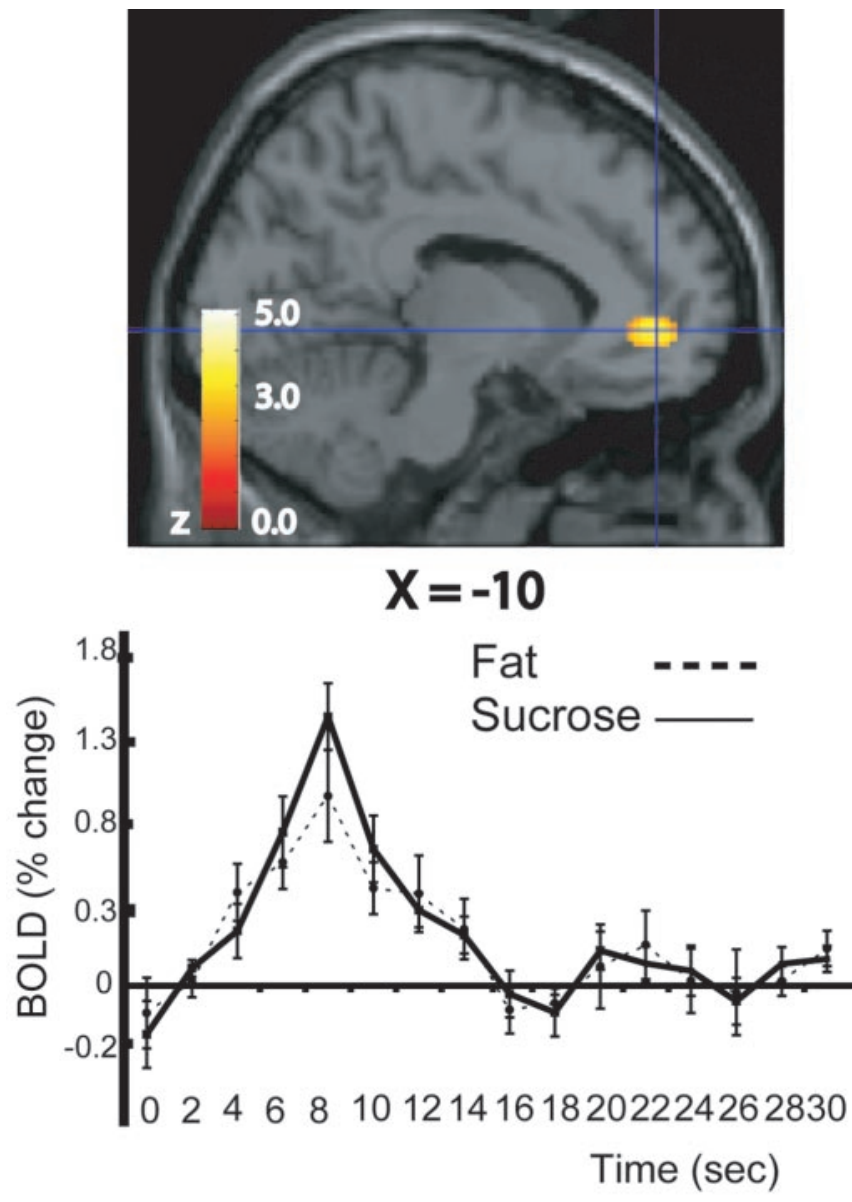

Figure 4. Top, Rostral anterior cingulate cortex activation by (fat - control) and (sucrose control), as revealed by conjunction analysis. Bottom, The corresponding average time course data (across trials and subjects) from the voxel marked by the crosshairs are shown.

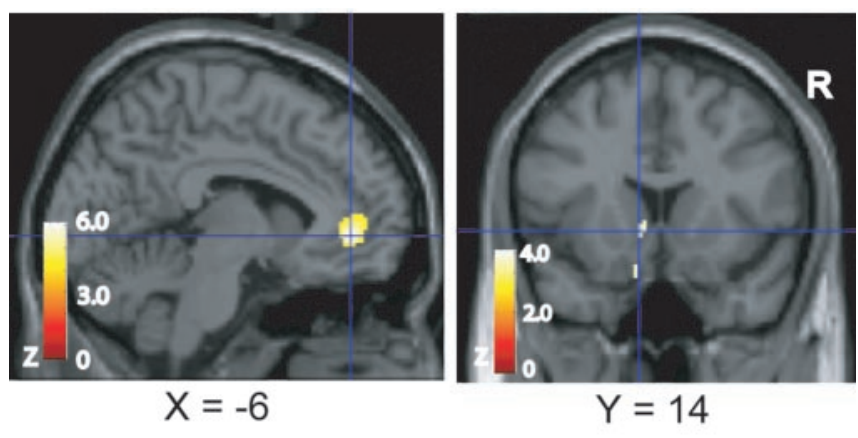

Figure 5. Activations correlating with stimulus fat content but independent of viscosity. Left, The main cluster of activation was observed in a rostral part of the anterior cingulate cortex, close to its transition zone with the medial orbitofrontal cortex. The parameter estimate for fat is $2.42 \pm 0.92$, for CMC at $50 \mathrm{CP}$ is $1.6 \pm 0.86$, and for CMC at $1000 \mathrm{CP}$ is $-0.14 \pm 0.31$. Right, In a region of interest analysis, activations were also observed in the ventral part of the left striatum. The parameter estimate for fat is $1.13 \pm 0.97$, for CMC at 50 cP is $0.73 \pm 0.62$, and for CMC at 1000 cP is $-0.13 \pm 0.85$. R, Right.

2003). However, activations by fat were also found in the anterior cingulate cortex and hypothalamus, and the anterior cingulate representation of fat was especially interesting, in that the activation here was independent of viscosity (produced by carboxymethyl cellulose). This anterior cingulate region was also activated by sucrose taste and is a strong candidate for a brain region activated by the hedonic properties of fat, of which it would be of interest given the present findings to investigate further in a satiety paradigm in which the hedonic properties of fat are reduced by feeding fat to satiety (cf. Kringelbach et al., 2003). Additional evidence linking this anterior cingulate region to pleasant affective properties (Bush et al., 2000) of sensory stimuli is that the same region is activated by water when it tastes pleasant during thirst (de Araujo et al., 2003b), by pleasant but not unpleasant odors (Rolls et al., 2003a), and by pleasant but not painful touch (Rolls et al., 2003c). Furthermore, this anterior cingulate region is also implicated in the control of autonomic function (Critchley et al., 2000a,b) and in putatively affective behavior such as vocalization (Devinsky et al., 1995). In addition, a number of other imaging studies have shown that the anterior cingulate cortex can be activated by hedonically relevant stimuli, including chemosensory stimuli (Zald and Pardo, 1997; Zald et al., 1998; Savic et al., 2000; Zatorre and Jones-Gotman, 2000; Zatorre et al., 2000; Small et al., 2001).

The areas activated by fat and by the viscosity stimuli described in this study were activated by texture and not by taste or olfactory stimulation in that care was taken to use a tasteless and odorless food bulking agent, carboxymethyl cellulose, for the viscosity series and to use the most odorless oil, a selected brand of vegetable oil, that we could locate. The psychophysical investigations reported in Results confirmed that the intensity of the taste of the carboxymethyl cellulose and vegetable oil was rated as low as that of water and very differently to the taste of $1 \mathrm{~m}$ sucrose. The same investigations confirmed that the intensity of the smell of the carboxymethyl cellulose and vegetable oil was rated as low as that of water and very differently from the intensity of the strawberry odor used in the neuroimaging investigation by de Araujo et al. (2003c), in which it was shown to activate olfactory cortical areas. Thus, the viscosity and oil stimulus used in this investigation were tasteless and odorless, and taste or olfactory stimulation can thus be discounted as contributing factors to the activations produced by these stimuli. In addition, we note that there is evidence that there is a taste and not olfactory part of the human primary taste cortex [which is quite anterior, with MNI $Y$ coordinate in the range of 3-14, and also lateral and dorsal, e.g., at $(-36,12,12)$ (de Araujo et al., 2003c), a finding consistent with single-neuron recordings in the macaque primary taste cortex Kadohisa et al., 2003], but that the part of the insula activated by taste and by viscosity in this study [Fig. 2, at MNI coordinates $(-36,12,6)]$ may have an olfactory input, as does the more anterior and ventral agranular insula (de Araujo et al., 2003c; Rolls et al., 2003a) (with additional studies showing that some parts of the insula can be activated by odor, including Small et al., 1997; Zald and Pardo, 1997; Cerf-Ducastel and Murphy, 2001; Poellinger et al., 2001; Anderson et al., 2003).

The ventral striatum is an important structure for rewardrelated learning and behavior and receives from the orbitofrontal cortex and amygdala (Rolls, 1999; Robbins and Everitt, 2002). Intra-accumbens administration of $\mu$-opioid agonists increases food intake and preferentially enhances the intake of palatable foods such as fat and sucrose (Kelley et al., 2002), and the intake of fat is influenced by activity in a neural system linking ventral striatal opioid systems with diencephalic and brainstem structures (Will et al., 2003). The fat-responsive cortical areas described in this study in humans and analyzed by single-neuron recording studies in macaques may provide the brain representation of the sensory properties of food in the mouth that are then linked to action by the striatal and brainstem systems to which we refer. 
In this investigation, the subjective ratings of oral fat were correlated with activations in both the anterior and mid-insular regions, in the orbitofrontal cortex, and in the anterior cingulate cortex. However, given that there was a moderate correlation between the subjective ratings of oral fat and thickness (0.75), the correlations with subjective fat ratings and activations in these brain regions were not exclusive and were also present for subjective ratings of thickness. Although textural signals related to viscosity may be one cue used to assess the fat content of food (Mela, 1988; Mela et al., 1994), we do not believe that this is the only texture channel used to signal the presence of fat in the mouth, in view of the single-neuron primate neurophysiological findings that some single neurons in the orbitofrontal cortex respond to fat but not to carboxymethyl cellulose and that other neurons respond to carboxymethyl cellulose but not to fat (Rolls et al., 1999, 2003b; Verhagen et al., 2003).

In conclusion, we showed for the first time some of the human brain systems activated by oral viscosity and fat. The insular and orbitofrontal areas may project into the cingulate, ventral striatal, and hypothalamic regions in which effects of intraoral fat were demonstrated. These findings pave the way for new investigations of where in the brain the palatability of fat in the mouth is represented, how different satiety signals affect these representations, and how these processes may differ in obese humans.

\section{References}

Anderson AK, Christoff K, Stappen I, Panitz D, Ghahremani DG, Glover G, Gabrieli JD, Sobel N (2003) Dissociated neural representations of intensity and valence in human olfaction. Nat Neurosci 6:196-202.

Bush G, Luu P, Posner MI (2000) Cognitive and emotional influences in anterior cingulate cortex. Trends Cogn Sci 4:215-222.

Cerf-Ducastel B, Murphy C (2001) fMRI activation in response to odorants orally delivered in aqueous solutions. Chem Senses 26:625-637.

Collins D, Neelin P, Peters T, Evans AC (1994) Automatic 3D intersubject registration of MR volumetric data in standardized Talairach space. J Comp Assist Tomogr 18:192-205.

Criqui MH, Heiss G, Cohn R, Cowan LD, Suchindran CM, Bangdiwala S, Kritchevsky S, Jacobs Jr DR, O'Grady HK, Davis CE (1993) Plasma triglyceride level and mortality from coronary heart disease. N Engl J Med 328:1220-1225.

Critchley HD, Elliott R, Mathias CJ, Dolan RJ (2000a) Neural activity relating to generation and representation of galvanic skin conductance responses: a functional magnetic resonance imaging study. J Neurosci 20:3033-3040.

Critchley HD, Corfield DR, Chandler MP, Mathias CJ, Dolan RJ (2000b) Cerebral correlates of autonomic cardiovascular arousal: a functional neuroimaging investigation in humans. J Physiol (Lond) 523:259-270.

de Araujo IE, Kringelbach ML, Rolls ET, Hobden P (2003a) Representation of umami taste in the human brain. J Neurophysiol 90:313-319.

de Araujo IE, Kringelbach ML, Rolls ET, McGlone F (2003b) Human cortical responses to water in the mouth, and the effects of thirst. J Neurophysiol 90:1865-1876.

de Araujo IE, Rolls ET, Kringelbach ML, McGlone F, Phillips N (2003c) Taste-olfactory convergence, and the representation of the pleasantness of flavour, in the human brain. Eur J Neurosci 18:2059-2068.

Devinsky O, Morrell MJ, Vogt BA (1995) Contributions of anterior cingulate cortex to behaviour. Brain 118:279-306.

Drewnowski A (1998) Energy density, palatability, and satiety: implications for weight control. Nutr Rev 56:347-353.

Francis S, Rolls ET, Bowtell R, McGlone F, O’Doherty J, Browning A, Clare S, Smith E (1999) The representation of pleasant touch in the brain and its relationship with taste and olfactory areas. NeuroReport 10:453-459.

Friston K, Jezzard P, Turner R (1994) Analysis of functional MRI timeseries. Hum Brain Mapp 1:153-171.

Friston KJ, Holmes AP, Price CJ, Buchel C, Worsley KJ (1999) Multisubject fMRI studies and conjunction analyses. NeuroImage 10:385-396.

Friston KJ, Glaser DE, Henson RN, Kiebel S, Phillips C, Ashburner J (2002)
Classical and Bayesian inference in neuroimaging: applications. NeuroImage 16:484-512.

Genovese CR, Lazar NA, Nichols T (2002) Thresholding of statistical maps in functional neuroimaging using the false discovery rate. NeuroImage 15:870-878.

Grafton ST, Woods RP, Mazziotta JC, Phelps ME (1991) Somatotopic mapping of the primary motor cortex in humans: activation studies with cerebral blood flow and positron emission tomography. J Neurophysiol 66:735-743.

Jain N, Qi HX, Catania KC, Kaas JH (2001) Anatomic correlates of the face and oral cavity representations in the somatosensory cortical area $3 \mathrm{~b}$ of monkeys. J Comp Neurol 429:455-468.

Kadohisa M, Verhagen JV, Rolls ET (2003) Taste, texture, and temperature but not olfactory or visual inputs to the primate taste cortex. Chem Senses 28:552.

Kelley AE, Bakshi VP, Haber SN, Steininger TL, Will MJ, Zhang M (2002) Opioid modulation of taste hedonics within the ventral striatum. Physiol Behav 76:365-377.

Kiebel SJ, Poline JB, Friston KJ, Holmes AP, Worsley KJ (1999) Robust smoothness estimation in statistical parametric maps using standardized residuals from the general linear model. NeuroImage 10:756-766.

Kringelbach ML, O’Doherty J, Rolls ET, Andrews C (2003) Activation of the human orbitofrontal cortex to a liquid food stimulus is correlated with its subjective pleasantness. Cereb Cortex 13:1064-1071.

Manger PR, Woods TM, Jones EG (1996) Representation of face and intraoral structures in area $3 \mathrm{~b}$ of macaque monkey somatosensory cortex. J Comp Neurol 371:513-521.

McCrory MA, Fuss PJ, McCallum JE, Yao M, Vinken AG, Hays NP, Roberts SB (1999) Dietary variety within food groups: association with energy intake intake and body fatness in men and women. Am J Clin Nutr 69:440-447.

Mela DJ (1988) Sensory assessment of fat content in fluid dairy products. Appetite 10:37-44.

Mela DJ, Langley KR, Martin A (1994) Sensory assessment of fat content: effect of emulsion and subject characteristics. Appetite 22:67-81.

O’Doherty J, Rolls ET, Francis S, Bowtell R, McGlone F (2001) Representation of pleasant and aversive taste in the human brain. J Neurophysiol 85:1315-1321.

Pardo JV, Wood TD, Costello PA, Pardo PJ, Lee JT (1997) PET study of the localization and laterality of lingual somatosensory processing in humans. Neurosci Lett 234:23-26.

Poellinger A, Thomas R, Lio P, Lee A, Makris N, Rosen BR, Kwong KK (2001) Activation and habituation in olfaction-an fMRI study. NeuroImage 13:547-560.

Robbins TW, Everitt BJ (2002) Limbic-striatal memory systems and drug addiction. Neurobiol Learn Mem 78:625-636.

Rolls ET (1997) Taste and olfactory processing in the brain and its relation to the control of eating. Crit Rev Neurobiol 11:263-287.

Rolls ET (1999) The brain and emotion. Oxford: Oxford UP.

Rolls ET (2000) The orbitofrontal cortex and reward. Cereb Cortex 10:284-294.

Rolls ET, Rolls BJ, Rowe EA (1983) Sensory-specific and motivationspecific satiety for the sight and taste of food and water in man. Physiol Behav 30:185-192.

Rolls ET, Critchley HD, Browning AS, Hernadi A, Lenard L (1999) Responses to the sensory properties of fat of neurons in the primate orbitofrontal cortex. J Neurosci 19:1532-1540.

Rolls ET, Kringelbach ML, de Araujo IE (2003a) Different representations of pleasant and unpleasant odours in the human brain. Eur J Neurosci 18:695-703.

Rolls ET, Verhagen JV, Kadohisa M (2003b) Representations of the texture of food in the primate orbitofrontal cortex: neurons responding to viscosity, grittiness, and capsaicin. J Neurophysiol 90:3711-3724.

Rolls ET, O’Doherty J, Kringelbach ML, Francis S, Bowtell R, McGlone F (2003c) Representations of pleasant and painful touch in the human orbitofrontal and cingulate cortices. Cereb Cortex 13:308-317.

Savic I, Gulyas B, Larsson M, Roland P (2000) Olfactory functions are mediated by parallel and hierarchical processing. Neuron 26:735-745.

Scott TR, Yaxley S, Sienkiewicz ZJ, Rolls ET (1986) Gustatory responses in the frontal opercular cortex of the alert cynomolgus monkey. J Neurophysiol 56:876-890. 
Small DM, Jones-Gotman M, Zatorre RJ, Petrides M, Evans AC (1997) Flavor processing: more than the sum of its parts. NeuroReport 8:3913-3917.

Small DM, Zatorre RJ, Dagher A, Evans AC, Jones-Gotman M (2001) Changes in brain activity related to eating chocolate: from pleasure to aversion. Brain 124:1720-1733.

Small DM, Zald DH, Jones Gotman M, Zatorre RJ, Pardo JV, Frey S, Petrides M (1999) Human cortical gustatory areas: a review of functional neuroimaging data. NeuroReport 10:7-14.

Theunissen MJ, Kroeze JH (1995) The effect of sweeteners on perceived viscosity. Chem Senses 20:441-450.

Thévenaz P, Blu T, Unser M (2000) Interpolation revisited. IEEE Trans Med Imaging 19:739-758.

Verhagen JV, Rolls ET, Kadohisa M (2003) Neurons in the primate orbitofrontal cortex respond to fat texture independently of viscosity. J Neurophysiol 90:1514-1525.

Will MJ, Franzblau EB, Kelley AE (2003) Nucleus accumbens $\mu$-opioids regulate intake of a high-fat diet via activation of a distributed brain network. J Neurosci 23:2882-2888.
Wilson J, Jenkinson M, de Araujo IET, Kringelbach ML, Rolls ET, Jezzard P (2002) Fast, fully automated global and local magnetic field optimization for fMRI of the human brain. NeuroImage 17:967-976.

Worsley KJ, Marrett P, Neelin AC, Friston KJ, Evans AC (1996) A unified statistical approach for determining significant signals in images of cerebral activation. Hum Brain Mapp 4:58-73.

Yaxley S, Rolls ET, Sienkiewicz ZJ (1990) Gustatory responses of single neurons in the insula of the macaque monkey. J Neurophysiol 63:689-700.

Zald DH, Pardo JV (1997) Emotion, olfaction, and the human amygdala: amygdala activation during aversive olfactory stimulation. Proc Natl Acad Sci USA 94:4119-4124.

Zald DH, Lee JT, Fluegel KW, Pardo JV (1998) Aversive gustatory stimulation activates limbic circuits in humans. Brain 121:1143-1154.

Zatorre RJ, Jones-Gotman M (2000) Functional imaging of the chemical senses. In: Brain mapping: the systems (Toga AW, Mazziotta JC, eds) New York: Academic.

Zatorre RJ, Jones-Gotman M, Rouby C (2000) Neural mechanisms involved in odor pleasantness and intensity judgments. NeuroReport 11:2711-2716. 